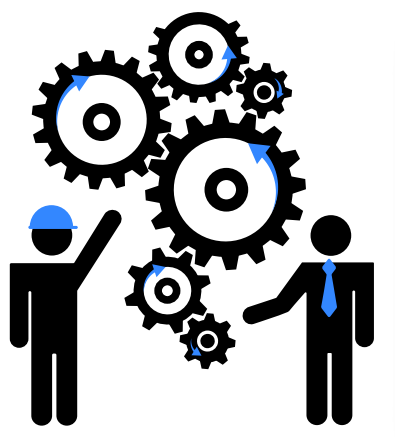

\title{
DISEÑO ESTRUCTURAL DE TANQUES RECTANGULARES Y SUS APLICACIONES
}

\author{
Structural design of rectangular tanks and applications
}

\author{
Jim Orihuela Canchari¹, Natividad A. Sánchez Arévalo² \\ 1 Bachiller en Ingeniería Civil, Universidad Continental \\ Correo electrónico: yeytijim10@hotmail.com \\ 2 Magíster en Ingeniería Civil, docente del curso Concreto Armado de la Universidad \\ Continental \\ Correo electrónico: nsanchez@continetal.edu.pe
}

Resumen. El presente artículo da a conocer el análisis y diseño de algunas de las múltiples aplicaciones de los tanques rectangulares contenedores de líquidos. El diseño de este tipo de tanques rectangulares debe garantizar, además de la adecuada resistencia, la durabilidad y la impermeabilidad para que pueda funcionar correctamente durante su vida útil. Para el análisis de las paredes de los tanques rectangulares, se emplearon metodologías basadas en el comportamiento estructural natural de las losas, unidireccional o bidireccional, las que dependen de las características geométricas y condiciones de borde en los apoyos del paño (empotrado, simplemente apoyado o combinación de estos) ante la acción de las cargas ejercidas por el agua, suelo y sobrecargas, las cuales pueden ser triangulares y/o uniformemente distribuidas.

Cuando se presenta el caso de comportamiento unidireccional, el análisis suele ser sencillo y accesible a cualquier método de análisis estructural. Sin embargo, en el caso de losas con comportamiento bidireccional, debe recurrirse a análisis basados en matemáticas avanzadas, por lo que es necesario el empleo de tablas que ofrecen resultados aproximados a la realidad, tales como: las tablas de la Asociación del Cemento Portland (PCA) [1], las tablas de Bares [2], entre otras. Para el diseño de las paredes del tanque rectangular, se ha verificado el correcto funcionamiento y se garantiza, fundamentalmente, la impermeabilidad, motivo por el cual se utilizó la metodología de diseño especificada en el ACI 350 [3], la cual toma en cuenta tres aspectos fundamentales: diseño por resistencia en etapa última; durabilidad y control de fisuras en etapa de servicio.

Para una mejor comprensión del presente, se desarrolla el diseño de un reservorio apoyado en el suelo.

Palabras clave: Resistencia; durabilidad; impermeabilidad; tablas de la PCA; tablas de Bares; control de fisuras.
Abstract. The present article shows the analysis and design of some of the multiple applications of rectangular liquid holding tanks. The design of said rectangular tanks must guarantee, beyond adequate resistance, durability and impermeability for the correct functioning during its whole use.

For the analysis of the water tank's walls, natural structural behavior based methodologies were used on the slabs, unidirectional and bidirectional, which depend on geometric properties and the sector's support conditions (recessed, simply supported or the combination of the before mentioned) by the action of the charge caused by water bodies, terrain and live charges, which can be triangular or uniformly distributed.

When the case of unidirectional behavior presents itself, the analysis tends to be simple and accessible to any method of structural analysis. Nevertheless, in case of slabs that presents bidirectional behavior, its necessary to execute advanced math based analysis, which demands the use of tables that offer approximate results, for example Portland Cement Association tables, Bares tables, among others.

For the design of the rectangular tank's walls it has been verified the correct functioning, guaranteeing primarily the impermeability, reason for which the specified design methodology of the ACI 350 [3], that considers three fundamental aspects: ultimate stage resistance design, durability and fissure control on service stage.

For better comprehension of the previous statements, a design for a ground supported reservoir will be developed.

Keywords: Resistance; Durability; Impermeability; PCA tables; Bares tables; fissure control. 


\section{INTRODUCCIÓN}

Los objetivos del presente artículo son los siguientes:

1. Dar a conocer las diversas aplicaciones de los tanques rectangulares de concreto armado.

2. Dar a conocer el proceso de análisis y diseño estructural de los tanques rectangulares de concreto armado.

Para dicho fin se desarrollan los siguientes temas:

- Aplicaciones de los tanques rectangulares en las obras civiles.

- Análisis estructural de los tanques rectangulares.

- Métodos de diseño para tanques rectangulares basados en la NTE E.o6o [4] y el Código Ambiental ACI 350.

- Ejemplo del diseño de un reservorio apoyado utilizando las tablas de la PCA y basado en las recomendaciones del $A C I 350$.

\section{APLICACIONES DE LOS TANQUES} RECTANGULARES

En el Perú y en otras partes del mundo, existe la necesidad de almacenamiento, abastecimiento, tratamiento y conducción de agua; las obras civiles que sirven para satisfacer aquellas necesidades, generalmente, son los tanques, los cuales deben tener un proceso constructivo económico, $100 \%$ resistentes, durables e impermeables. Esto se consigue mediante el empleo de métodos de diseño, basados en el Código del Medio Ambiente ACI-350 [3]. Uno de los materiales de construcción que garantiza los requisitos que anteceden para el logro de tanques funcionales, y que además existe disponibilidad de mano de obra por su fácil manejo constructivo, es el concreto armado [5].

Los tanques rectangulares cumplen múltiples funciones en diversas obras de la ingeniería civil [3], las cuales contribuyen a mejorar el medio ambiente, satisfacer necesidades requeridas por los seres humanos, animales y plantas, como se mencionan a continuación:

- Almacenamiento y abastecimiento de agua potable: tanques superficiales (reservorios apoyados), tanques elevados, tanques cisterna.

- Almacenamiento y abastecimiento para riego: reservorios de almacenamiento de agua para riego.

- Con fines recreativos-deportivos: piscinas.
- Con fines productivos: estanques para cultivo de peces.

- Tratamiento de agua potable: plantas potabilizadoras.

- Tratamiento de aguas residuales: desarenadores, sedimentadores, tanque imhoff, lecho de secado, laguna de estabilización, tanque séptico.

- Conducción de aguas: puente canal, conducto cubierto.

Los tanques rectangulares se limitan a volúmenes que permitan obtener espesores económicos de sus elementos, porque sus componentes (paredes, fondo y/o tapa) trabajan a flexión y fuerza cortante, motivo por el cual se requiere que las dimensiones sean lo suficiente como para garantizar rigidez a flexión y resistencia a fuerza cortante. Cuando se sobrepasan estos espesores, será recomendable utilizar tanques con elementos curvos tipo cáscara y/o estructuras laminares, los cuales basan su comportamiento estructural en esfuerzos axiales de tracción y/o compresión, lo que permite obtener secciones de menor espesor (por ej., tanques cilíndricos o tanques intze que sirven para almacenar grandes volúmenes de agua).

\section{ANÁLISIS ESTRUCTURAL DE LOS TANQUES RECTANGULARES}

El análisis de las paredes de los tanques rectangulares se hace teniendo en cuenta las metodologías basadas en el comportamiento estructural de las losas, unidireccional o bidireccional, las cuales dependen de las características geométricas y condiciones de borde en los apoyos del paño (empotrado, simplemente apoyado o una combinación de estos) ante la acción de las cargas estáticas ejercidas por la presión del agua, presión del suelo, cuando los tanques sean enterrados (véase Figura 1), y sobrecargas, que pueden ser triangulares y/o uniformemente distribuidas.

Para el caso de losas unidireccionales, el análisis se efectúa aplicando las metodologías clásicas existentes; en el caso de las losas bidireccionales, cuyo análisis es más complicado, se emplean tablas cuyos resultados son aproximados a la realidad, tales como: PCA, Bares, Kalmanok, entre otras.

Para el desarrollo del presente artículo, se aplican las tablas de la PCA, que son más fáciles de emplear.

Para el caso de zonas sísmicas, se analizarán los efectos hidrodinámicos del agua en interacción con los 

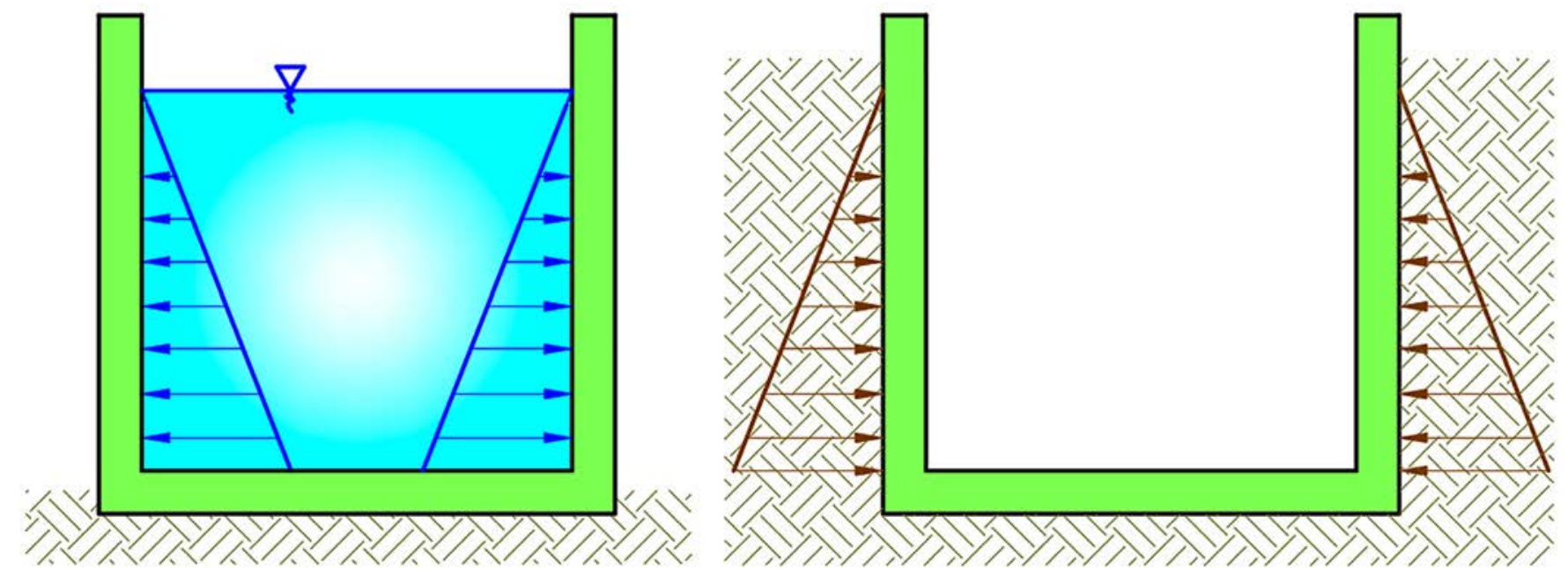

Figura 1. Presiones de agua y presiones de suelo
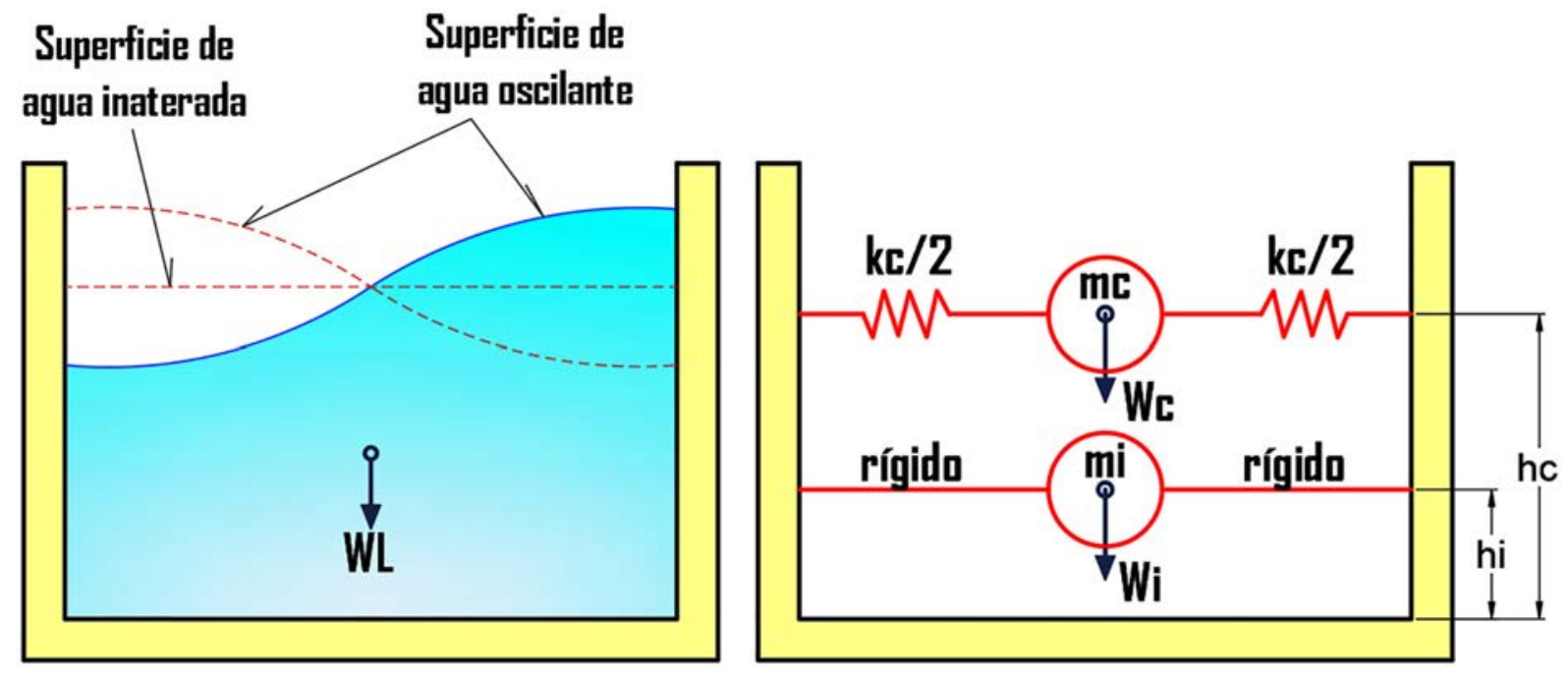

Figura 2. Sistema dinámico equivalente para tanques de agua

componentes del tanque (paredes, fondo y tapa), aun cuando Harmsen [5] y Pavón [6] no consideran relevante la acción de las fuerzas sísmicas en tanques rectangulares de dimensiones limitadas, como es usual.

George W. Housner [7] define a la masa de agua en movimiento como masa impulsiva (mi) y masa convectiva (mc). La masa impulsiva es la que se mueve en conjunto con la estructura; la masa convectiva es producida por la oscilación del agua (véase Fig. 2).

Las fuerzas impulsivas (Wi) y convectivas (Wc) se determinan con las expresiones desarrolladas por Housner, las cuales se encuentran también en el $A C I$ $350.3[8]$.

$$
\begin{array}{r}
\mathbf{W}_{\mathbf{L}}=\mathrm{W}_{\mathrm{L}}\left[\frac{\tanh \left(0.866 \frac{\mathrm{L}}{\mathrm{L}_{\mathrm{L}}}\right)}{0.866 \frac{\mathrm{L}}{\mathrm{H}_{\mathrm{L}}}}\right] \\
\mathbf{W}_{\mathrm{c}}=\mathbf{W}_{\mathrm{L}}\left[0.264\left(\frac{\mathrm{L}}{\mathrm{H}_{\mathrm{L}}}\right) \tanh \left(3.16 \frac{\mathrm{H}_{\mathrm{L}}}{\mathrm{L}}\right)\right]
\end{array}
$$

Con Wi y Wc se determinan las presiones hidrodinámicas en la base de las paredes del tanque, cuyo procedimiento se muestra en el ejemplo de diseño ítem 5.3. Cabe mencionar que el tema tratado se limita a los tanques rectangulares superficiales y/o enterrados.

\section{MÉTODOS DE DISEÑO}

\subsection{Método de diseño por resistencia [3]}

Según la NTE E.o6o, el Método de Diseño por Resistencia (Artículo 9.1.1) es el recomendado para diseñar estructuras de concreto armado.

Artículo 9.1.1. Las estructuras y elementos estructurales deberán diseñarse para obtener en todas sus secciones resistencias de diseño $(\phi R n)$ por 
lo menos iguales a las resistencias requeridas $(\mathrm{Ru})$, calculadas para las cargas y fuerzas amplificadas en las combinaciones que se estipulan en esta Norma. En todas las secciones de los elementos estructurales deberá cumplirse:

$$
\phi R n \geq R u
$$

Donde $\phi$ es el factor de reducción de resistencia, menor que la unidad, que corresponda.

1. Flexión, sin carga axial .......... $\phi=0,90$
2. Cortante y torsión .............. $\phi=0,85$

Aunque en la Norma Técnica E.o6o no existe un capítulo con especificaciones para el diseño de tanques, se han considerado las disposiciones del Artículo 9.2 (Resistencia requerida) y se complementa con las disposiciones del Código Ambiental ACI 350 para el diseño de estructuras contenedoras de líquidos, el cual recomienda procedimientos para el uso combinado del método de esfuerzos admisibles, con el método de la resistencia.

El ACI 350 incrementa las cargas últimas (factorizadas) obtenidas con el método de resistencia (E.o6o), multiplicándolas por un coeficiente de durabilidad sanitaria «S», que, dependiendo de los esfuerzos, tiene los siguientes valores:

$$
\begin{aligned}
& S=1.3 \text {, para flexión } \\
& S=1.65 \text {, para tracción directa } \\
& S=1.3 \text {, para cortante }
\end{aligned}
$$

De esta manera, los factores de carga para el diseño estructural de los tanques son los siguientes:

a) Para elementos estructurales sometidos a carga muerta $(\mathrm{CM})$ y a carga viva $(\mathrm{CV})$, la resistencia requerida es $\mathrm{U}=1.3 \times(1.4 \mathrm{CM}+1.7 \mathrm{CV})$.

b) Para estructuras sometidas a empujes de suelo (CE), la resistencia requerida es

$$
\mathrm{U}=1.3 \times(1.4 \mathrm{CM}+1.7 \mathrm{CV}+1.7 \mathrm{CE}) \text {. }
$$

c) Para estructuras sometidas a presión de líquidos (CL), la resistencia requerida (según E.o6o) es $\mathrm{U}=1.4 \mathrm{CM}+1.7 \mathrm{CV}+1.4 \mathrm{CL}$; sin embargo, para CL, el ACI 350 recomienda emplear el factor 1.7 en lugar de 1.4. Entonces la combinación de carga cuando haya presión de líquidos será:

$\mathrm{U}=1.3 \times(1.4 \mathrm{CM}+1.7 \mathrm{CV}+1.7 \mathrm{CL})$

Para el caso de fuerzas sísmicas, el ACI 350 nos muestra las siguientes combinaciones, las cuales son afectadas también por el coeficiente de durabilidad sanitaria:

$$
\begin{gathered}
U=1.3 \times(0.9 \mathrm{D}+1.2 \mathrm{~F}+1.0 \mathrm{E}+1.6 \mathrm{H}) \\
\mathrm{U}=1.3 \times(1.2 \mathrm{D}+1.2 \mathrm{~F}+1.0 \mathrm{E}+1.6 \mathrm{H}+1.0 \mathrm{~L}+0.2 \mathrm{~S})
\end{gathered}
$$

Donde: $\mathrm{D}=$ carga muerta, $\mathrm{F}$ = presión estática del fluido, $\mathrm{E}=$ cargas de sismo, $\mathrm{H}$ = presión del suelo, $\mathrm{L}=$ carga viva, $\mathrm{S}=$ carga de nieve.

\subsection{Verificación del correcto funcionamiento del tanque bajo cargas de servicio (control de fisuras)}

Los resultados obtenidos con el método de diseño por resistencia deberán ser verificados bajo cargas de servicio, según la NTE E.o6o

Artículo 9.1.2 Las estructuras y los elementos estructurales deberán cumplir además con todos los demás requisitos de esta Norma, para garantizar un comportamiento adecuado bajo cargas de servicio.

Para prevenir fugas y garantizar la impermeabilidad que requieren los tanques, el Código Ambiental ACI 350 recomienda realizar el control de fisuras en términos de que el ancho de estas sea el mínimo posible. Este control se consigue proporcionando una adecuada distribución y verificando el espaciamiento máximo «S» de los aceros de refuerzo. Este espaciamiento máximo se evalúa en la etapa elástica, bajo cargas de servicio, y se calcula con la siguiente ecuación:

$$
S_{\max }=\frac{Z^{3}}{2{d c^{2}}^{2} s^{3}}
$$

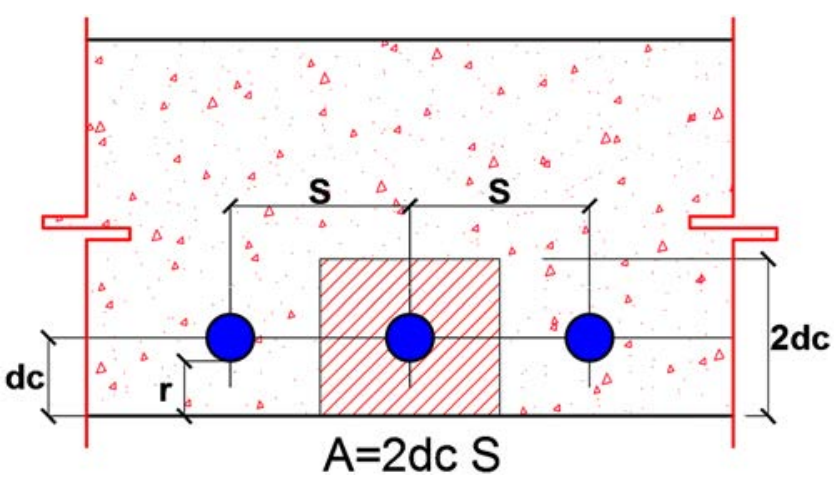

Figura 3. Esquema para el cálculo del espaciamiento máximo para control de fisuras

Donde:

$$
\mathrm{Z}=\mathrm{fs} \sqrt[3]{\mathrm{dcA}}
$$

$\mathrm{S}$ = separación de las varillas en $\mathrm{cm}$

$\mathrm{A}=2 \mathrm{dcS}$

$\mathrm{dc}=$ recubrimiento del concreto medido desde la fibra extrema de tensión, al centro de la varilla en $\mathrm{cm}$.

fs = esfuerzo en el acero en condiciones de servicio, en $\mathrm{kg} / \mathrm{cm}^{2}$

$$
\mathrm{fs}=\frac{\mathrm{Ms}}{0.9 \mathrm{~d} \mathrm{As}}
$$


$\mathrm{Ms}=$ momento flector en condiciones de servicio

As $=$ acero colocado

$\mathrm{d}=$ Peralte efectivo (en función del recubrimiento «r»)

Los valores de $\mathbf{Z}$, según el $A C I$ 350, están en función a la exposición al ambiente:

- Para condiciones normales $Z \leq 20580 \mathrm{~kg} / \mathrm{cm}$, que corresponde a rajaduras de $0,025 \mathrm{~cm}$.

- Para condiciones severas $Z \leq 17000 \mathrm{~kg} / \mathrm{cm}$, que corresponde a rajaduras de $0,020 \mathrm{~cm}$.

Tabla 1. Recubrimientos mínimos y peralte efectivo

\begin{tabular}{|l|c|c|}
\hline \multicolumn{1}{|c|}{ Elemento } & $\mathbf{r}(\mathbf{c m})$ & $\mathbf{d}(\mathbf{c m})$ \\
\hline Paredes & 5,0 & e-6 \\
\hline $\begin{array}{l}\text { Zapatas y losas de fondo en } \\
\text { contacto con el suelo }\end{array}$ & 7,5 & h-10 \\
\hline $\begin{array}{l}\text { Parte superior de zapatas y losas } \\
\text { de fondo }\end{array}$ & 5,0 & h-6 \\
\hline \begin{tabular}{l} 
Losa de tapa \\
\hline
\end{tabular} & 5,0 & e-6 \\
\hline
\end{tabular}

V. EJEMPLO DE DISEÑO DE UN RESERVORIO APOYADO CON LAS TABLAS DE LA PCA

Se diseña un reservorio con tapa, apoyado sobre la superficie del terreno, cuya función es el almacenamiento de agua potable y tiene las siguientes características:

- Dimensiones internas en planta: 4,5 x 4,5 m.

- Altura: 3,00 metros

- Capacidad: $50 \mathrm{~m}^{3}$.

5.1. Dimensionamiento de las paredes y losa de tapa del reservorio

Luz larga $/$ luz corta $=4.5 / 3.0=1.5<2=>$ Las paredes tienen comportamiento bidireccional. Entonces, el espesor mínimo (e) de las paredes del reservorio, según $A C I$ 350, es:

- $\mathrm{e}=1 / 30=3 / 30=0.10 \mathrm{~m}$

- $h \geq 3.0 \mathrm{~m}=>\mathrm{e}_{\min }=0.30 \mathrm{~m}$

Como las paredes del reservorio tienen una altura de $3 \mathrm{~m}$, se ha utilizado el espesor mínimo de $\mathbf{e}=\mathbf{0 . 3 0} \mathbf{~ m}$ de acuerdo a la consideracion del ACI 350 (Artículo 14.6.2).

De acuerdo al dimensionamiento, las dimensiones del reservorio son las que se muestran en la Figura 4.

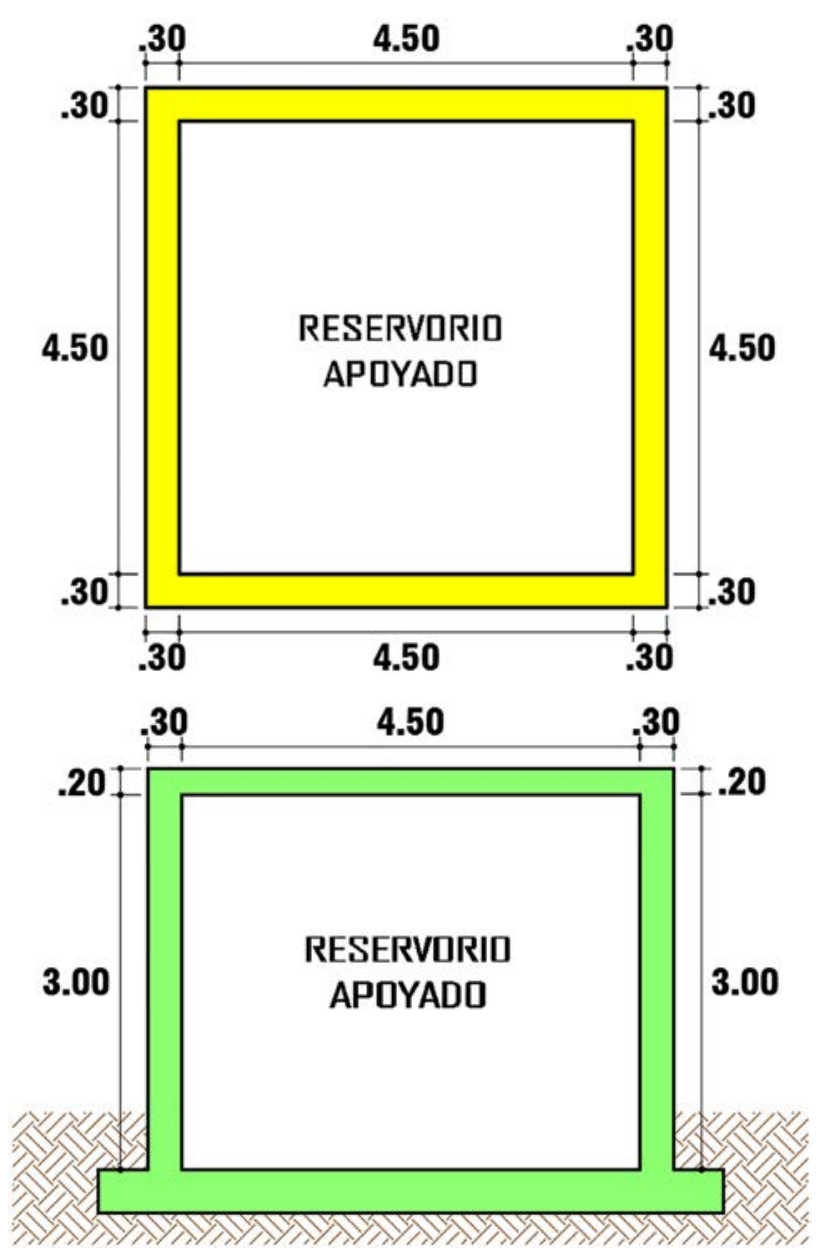

Figura 4. Dimensiones del reservorio en planta y en elevación

5.2. Determinación de las condiciones de borde en las paredes y losa de tapa del reservorio

El tanque está conformado por cuatro muros continuos entre sí; sus bordes laterales y el borde inferior con su cimentación son empotrados (ver Fig. 5).

Para el caso del borde superior, se verifican las condiciones de apoyo, teniendo en cuenta la relación de rigidez entre las paredes y la tapa. La expresión recomendada por el $A C I$ para evaluar las condiciones de apoyo, pared-losatapa, mediante la relación de rigideces a flexión ( $\left.\mathrm{K}_{\text {pared }} / \mathrm{K}_{\text {tapa }}\right)$, se determina como sigue:

- Kpared / Ktapa > $8 \rightarrow$ Empotrado

- Kpared / Ktapa $<8 \rightarrow$ Simplemente apoyado

Donde:

$\mathrm{K}=4 \mathrm{EI} / \mathrm{L}$;

$\mathrm{E}=$ módulo de elasticidad del concreto;

$\mathrm{I}=\mathrm{bh}^{3 / 12}$,

$\mathrm{h}=$ espesor de pared o losa tapa,

$\mathrm{L}=$ longitud del elemento. 


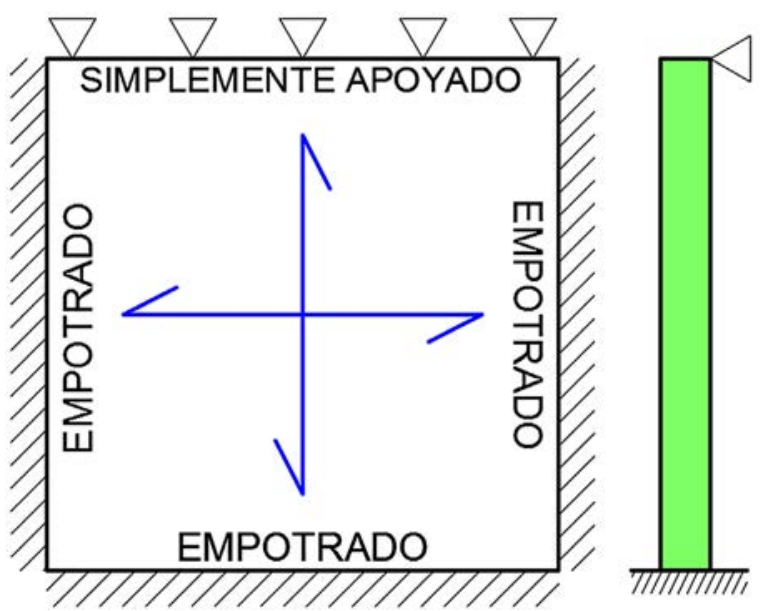

Figura 5. Condiciones de borde de las paredes y tapa del reservorio

El momento de inercia (I) se calcula para una sección de elemento de ancho $\mathrm{b}=1 \mathrm{~m}$.

La relación de rigideces $\mathrm{K}_{\text {pared }} / \mathrm{K}_{\text {tapa }}=5.27<8$. Entonces, la losa de tapa se considera simplemente apoyada en las paredes del reservorio (véase Fig. 5); sin embargo, le adicionaremos en el apoyo un momento de monolitismo de wul²/16 (Artículo 8.3.4 de la E.06o).

Tabla 2. Cálculo de la relación de rigideces entre la pared y la tapa del reservorio

\begin{tabular}{|l|c|c|c|}
\hline \multicolumn{1}{|c|}{ Elemento } & I & K & Kpared / Ktapa \\
\hline Pared & 225000 & 731,71 & \\
\hline Tapa & 66666,67 & 138,89 & 5,27 \\
\hline
\end{tabular}

5.3. Condiciones de carga para el análisis de las paredes del reservorio

\section{Condición de carga única: Prueba para fugas}

Como se trata de un reservorio superficial apoyado en el suelo, solo se considera la condición de carga más crítica cuando el tanque está completamente lleno. Esta consideración puede presentarse en cualquiera de los dos casos: 1) Para verificar la presencia de fugas del líquido durante el proceso constructivo y 2) Ante la probabilidad de ocurrencia de que se malogre el flotador del tanque.

Presión estática del agua (distribución triangular):

$$
\mathrm{q}_{1}=\gamma \mathrm{H}=1000 \mathrm{~kg} / \mathrm{m} 3 \times 3.00 \mathrm{~m}=3000 \mathrm{~kg} / \mathrm{m} 2
$$

La presión dinámica del agua se determina mediante las expresiones de cálculo desarrolladas por Housner y explicadas también en el $A C I$ 350.3.

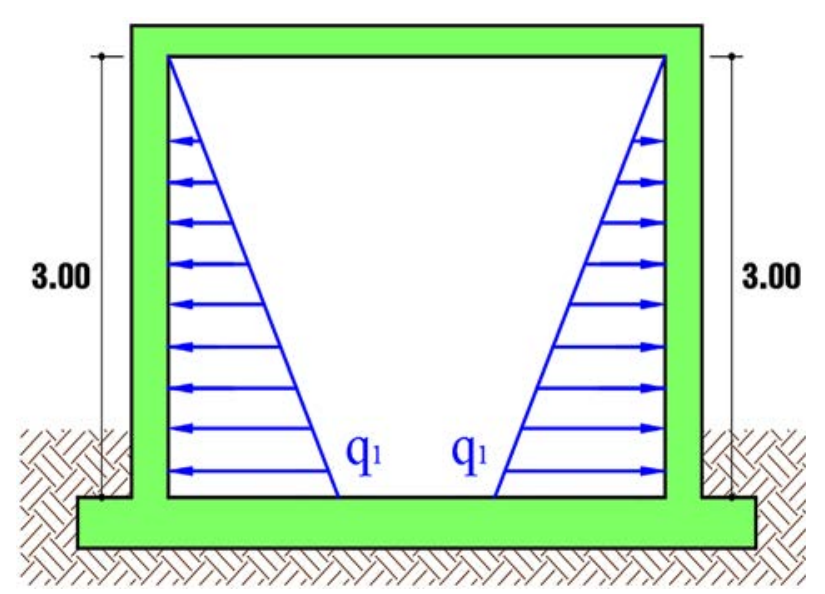

Figura 6. Condición de carga única para el diseño del reservorio

Las medidas eje a eje del reservorio, según el dimensionamiento, son:

- $\mathrm{Lx}=\mathrm{Ly}=\mathrm{B}=4,80 \mathrm{~m}$

- Altura de los muros $\mathrm{H}=3,00 \mathrm{~m}$

- Espesor de los muros $\mathrm{tw}=0,20 \mathrm{~m}$

- Altura del líquido $\mathrm{H}_{\mathrm{L}=2,70 \mathrm{~m}}$

- Borde libre $=0,30 \mathrm{~m}$

Teniendo en cuenta el sistema dinámico equivalente para tanques de agua propuesto por Housner (véase Fig. 2), las fuerzas dinámicas se determinan de la siguiente manera:

$$
\begin{aligned}
& \boldsymbol{W}_{\boldsymbol{i}}=W_{L}\left[\frac{\tanh \left(0.866 \frac{\mathrm{L}}{H_{L}}\right)}{0.866 \frac{L}{H_{L}}}\right]=36852.54 \mathrm{~kg} \\
& \boldsymbol{W}_{\boldsymbol{c}}=W_{L}\left[0.264\left(\frac{L}{H_{L}}\right) \tanh \left(3.16 \frac{H_{L}}{L}\right)\right]=27573.72 \mathrm{~kg}
\end{aligned}
$$

Las fuerzas dinámicas laterales se determinan con los valores sísmicos: factor de zona (Huancayo) $\mathrm{Z}=$ 0.35 , factor de suelo $S=1$, factor de importancia $I=$ 1.3, factor de modificación de respuesta $\mathrm{R}_{\mathrm{wi}}=2,75 \mathrm{y}$ $\mathrm{R}_{\mathrm{wc}}=1,0$, factor de amplificación sísmica $\mathrm{Ci}=0,60 \mathrm{y}$ $\mathrm{Cc}=0,40$ :

- Fuerza producida por masa impulsiva

$$
\begin{aligned}
\mathrm{P}_{\mathrm{i}} & =\text { ZSIC }_{\mathrm{i}} \mathrm{x} \frac{\mathrm{W}_{i}}{\mathrm{R}_{\mathrm{wi}}} \\
\mathrm{P}_{\mathrm{i}} & =0.35 \times 1.0 \times 1.3 \times 0.60 \times \frac{36852.54 \mathrm{~kg}}{2.75} \\
\mathrm{P}_{\mathrm{i}} & =3658.45 \mathrm{~kg}
\end{aligned}
$$

- Fuerza producida por masa convectiva

$$
\begin{aligned}
\mathrm{P}_{\mathrm{c}} & =\operatorname{ZSIC}_{c} \times \frac{\mathrm{W}_{\mathrm{c}}}{\mathrm{R}_{\mathrm{wc}}} \\
\mathrm{P}_{\mathrm{c}} & =0.35 \times 1.0 \times 1.3 \times 0.40 \times \frac{27573.72 \mathrm{~kg}}{1} \\
\mathrm{P}_{\mathrm{c}} & =5018.42 \mathrm{~kg}
\end{aligned}
$$


La presión en la base de los muros se determina en la Figura 7.

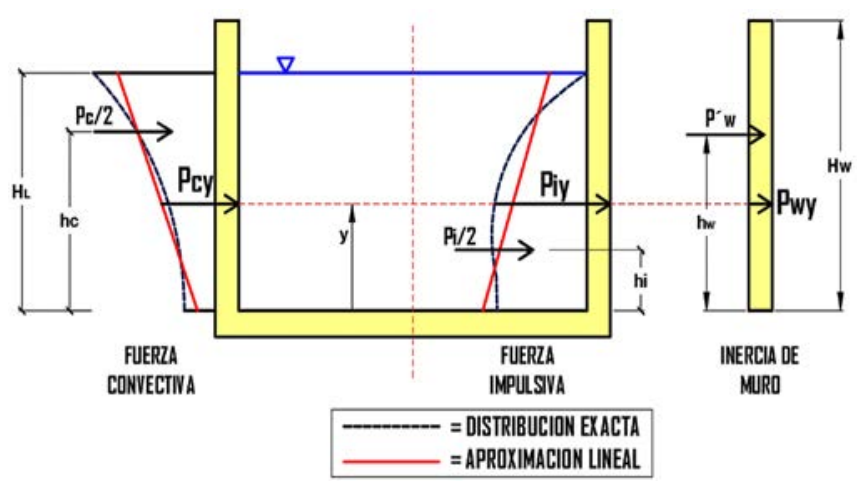

Figura 7. Fuerzas dinámicas en el tanque

Presión dinámica convectiva unitaria

$\mathrm{P}_{c y}=\frac{0.5 \mathrm{P}_{\mathrm{c}}\left[4 \mathrm{H}_{\mathrm{L}}-6 \mathrm{~h}_{\mathrm{c}}-\left(6 \mathrm{H}_{\mathrm{L}}-12 \mathrm{~h}_{\mathrm{c}}\right)\left(\frac{\mathrm{y}}{\mathrm{H}_{\mathrm{L}}}\right)\right]}{\mathrm{H}_{\mathrm{L}}{ }^{2}}=371.03 \mathrm{~kg} / \mathrm{m}$

$p_{c(y=0)}=\frac{P_{c y}}{B}=\frac{371.03}{4.8}=77.30 \mathrm{~kg} / \mathrm{m} 2$

\section{Presión dinámica impulsiva unitaria}

$P_{i y}=\frac{0.5 P_{i}\left[4 \mathrm{H}_{\mathrm{L}}-6 \mathrm{~h}_{\mathrm{c}}-\left(6 \mathrm{H}_{\mathrm{L}}-12 \mathrm{~h}_{\mathrm{c}}\right)\left(\mathrm{y} / \mathrm{H}_{\mathrm{L}}\right)\right]}{\mathrm{H}_{\mathrm{L}}{ }^{2}}=1185.61 \mathrm{~kg} / \mathrm{m}$

$p_{i(y=0)}=\frac{P_{i y}}{B}=\frac{1185.71}{4.8}=247.02 \mathrm{~kg} / \mathrm{m} 2$

Fuerza de inercia unitaria

$\mathrm{P}_{\mathrm{wy}}=\frac{\mathrm{ZSI}_{\mathrm{e}} \mathrm{C}_{\mathrm{i}}}{\mathrm{R}_{\mathrm{wi}}}\left(\varepsilon \gamma_{\mathrm{c}} \mathrm{B} \mathrm{t}_{\mathrm{w}}\right) / 12=20.87 \mathrm{~kg} / \mathrm{m}$

$p_{w y}=\frac{\mathrm{P}_{\mathrm{wy}}}{\mathrm{B}}=4.35 \mathrm{~kg} / \mathrm{m} 2$

Presión hidrodinámica por efecto de la aceleración vertical:

$p_{v y}=\ddot{\mathrm{u}}_{v} q_{h y}$

$\ddot{\mathrm{u}}_{v}=0.4 S_{D S}\left(\frac{2 / 3}{R_{i}}\right) \geq 0.2 S_{D S}=0.12$

$\mathrm{q}_{\mathrm{hy}}=\gamma\left(\mathrm{H}_{\mathrm{L}}-\mathrm{y}\right)=1000(2.7-0)=2700 \mathrm{~kg} / \mathrm{m} 2$

Entonces: $\mathrm{p}_{\mathrm{vy}}=0.12 \times 2700=324 \mathrm{~kg} / \mathrm{m} 2$

La presión hidrodinámica total (Py) en la base se determina con el método de la raíz cuadrada de la suma de los cuadrados:

$P_{y}=\sqrt{\left(P_{i y}+P_{w y}\right)^{2}+P_{c y}^{2}+P_{v y}^{2}}$

$\mathrm{P}_{\mathrm{y}}=\sqrt{(247.02+4.35)^{2}+77.30^{2}+324^{2}}=\mathbf{4 1 7 . 2 9} \mathrm{kg} / \mathrm{m} 2$

Una vez calculadas las presiones estáticas y dinámicas, pasamos a determinar las cargas últimas utilizando las combinaciones de carga mostradas en el ítem 3.1.
Para cargas estáticas, tenemos:

$\mathrm{U}=1.7 \mathrm{CL}$

$\mathrm{U}=1.7 \times 3000=5100 \mathrm{~kg} / \mathrm{m}^{2}$

Para cargas dinámicas, tenemos:

$\mathrm{U}=1.2 \mathrm{~F}+1.0 \mathrm{E}$

$\mathrm{U}=1.2 \times 3000+417.29=4017.29 \mathrm{~kg} / \mathrm{m}^{2}$

Como podemos ver la carga dinámica última es menor a la carga estática última, lo cual significa que las fuerzas sísmicas no alterarán el comportamiento de la estructura. Por lo tanto, el diseño del tanque se realizará para la acción de cargas estáticas.

\subsection{Diseño por flexión de las paredes del reser- vorio para la condición de carga única}

Para el diseño por flexión, se calculan los momentos flectores que se generan en las paredes del tanque, tanto en la dirección vertical como en la horizontal, para ello utilizamos los coeficientes de la Tabla 3, que corresponden al Caso 4 de la PCA, el cual corresponde a las condiciones de borde: 1) simplemente apoyado en el borde superior y empotrado en los bordes laterales y en la cimentación. Para ingresar a estas tablas, se requiere definir las relaciones de dimensiones largo/alto (b/a) y ancho/ alto (c/a), correspondiente a cada uno de los lados que conforman las paredes del tanque. Para el diseño del reservorio, las paredes tienen dimensiones iguales para los lados largo y corto (planos X-Y e Y-Z respectivamente), es decir $\mathrm{b}=\mathrm{c}$, se obtienen así relaciones $\mathrm{b} / \mathrm{a}=\mathrm{c} / \mathrm{a}=1.6$. Como las tablas de la PCA no presentan valores para estas relaciones, después de un análisis de la variación de estas tablas, para las relaciones especificadas en ellas, se ha optado por elegir a las que corresponden a relaciones de lados $\mathrm{b} / \mathrm{a}=2.0^{*}$ y $\mathrm{c} / \mathrm{a}=1.5^{1}$.

\section{Caso 4}

Borde superior simplemente apoyado, bordes laterales y fondo empotrados

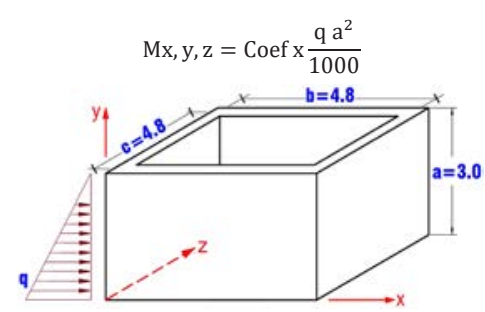
$\frac{b}{a}=2 \quad \frac{c}{\mathrm{a}}=1.5$

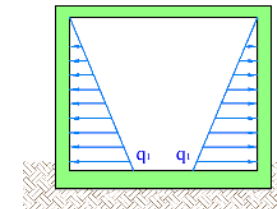

1 Tablas mostradas en el capítulo 3 de la PCA, página 3-41. 
Tabla 3. Coeficientes para el cálculo de momentos flectores en las paredes del reservorio (PCA)

\begin{tabular}{|c|c|c|c|c|c|c|c|c|c|c|c|c|c|c|c|c|c|c|c|}
\hline & \multicolumn{6}{|c|}{ Coeficientes para Mx } & \multicolumn{6}{|c|}{ Coeficientes para My } & \multicolumn{6}{|c|}{ Coeficientes para Mxy } \\
\hline & & Esq. & $\begin{array}{c}\mathbf{0 , 1 b} \\
\mathbf{0 , 9 b}\end{array}$ & $\begin{array}{l}\mathbf{0 , 2 b} \\
\mathbf{0 , 8 b}\end{array}$ & $\begin{array}{l}\mathbf{0 , 3 b} \\
\mathbf{0 , 7} \mathbf{b}\end{array}$ & $\begin{array}{c}0,4 b \\
0,6 b\end{array}$ & $0.5 \mathbf{b}^{*}$ & Esq. & $\begin{array}{c}\mathbf{0 , 1 b} \\
\mathbf{0 , 9 b}\end{array}$ & $\begin{array}{c}\mathbf{0 , 2 b} \\
\mathbf{0 , 8 b}\end{array}$ & $\begin{array}{l}0,3 b \\
0,7 b\end{array}$ & $\begin{array}{l}0,4 \mathbf{b} \\
\mathbf{0 , 6 b}\end{array}$ & $\mathbf{0 , 5 b}$ & Esq. & $\begin{array}{c}\mathbf{0 , 1 b} \\
\mathbf{0 , 9 b}\end{array}$ & $\begin{array}{l}\mathbf{0 , 2 b} \\
\mathbf{0 , 8 b}\end{array}$ & $\begin{array}{l}0,3 b \\
0,7 b\end{array}$ & $\begin{array}{c}0,4 b \\
0,6 b\end{array}$ & $\mathbf{0 , 5 b}$ \\
\hline \multirow{11}{*}{ 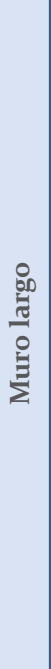 } & $\begin{array}{l}\text { Borde } \\
\text { sup. }\end{array}$ & o & o & o & o & o & o & o & o & o & o & o & o & 1 & 9 & 9 & 6 & 3 & o \\
\hline & $\mathbf{0 , 9 a}$ & -2 & 1 & 4 & 6 & 8 & 8 & -11 & -1 & 2 & 3 & 3 & 3 & 1 & 9 & 8 & 5 & 3 & o \\
\hline & $\mathbf{0 , 8 a}$ & -4 & 2 & 8 & 12 & 15 & 16 & -20 & -2 & 4 & 6 & 6 & 6 & 1 & 7 & 7 & 4 & 2 & o \\
\hline & $0,7 a$ & -6 & 3 & 11 & 17 & 21 & 22 & -28 & -3 & 6 & 8 & 8 & 8 & o & 5 & 5 & 3 & 1 & o \\
\hline & $0,6 a$ & -7 & 4 & 14 & 21 & 24 & 25 & -34 & -3 & 7 & 10 & 10 & 9 & o & 2 & 2 & 1 & o & o \\
\hline & $0,5 a^{* *}$ & -7 & 5 & 15 & 22 & 25 & 26 & -36 & -3 & 8 & 10 & 10 & 9 & o & 1 & 1 & 1 & 1 & o \\
\hline & $0,4 a$ & -7 & 5 & 15 & 22 & 25 & 26 & -34 & -2 & 7 & 9 & 8 & 8 & o & 4 & 4 & 3 & 1 & o \\
\hline & $0,3 a$ & -6 & 4 & 10 & 12 & 13 & 13 & -28 & -1 & 6 & 6 & 5 & 5 & o & 7 & 7 & 4 & 2 & o \\
\hline & $0,2 a$ & -4 & 1 & 1 & -1 & -2 & -3 & -18 & o & 3 & 2 & 1 & 1 & 1 & 8 & 7 & 4 & 2 & o \\
\hline & $\mathbf{0 , 1 a}$ & -1 & -6 & -15 & -22 & -26 & -27 & -6 & -1 & -2 & -4 & -5 & -5 & 1 & 7 & 5 & 3 & 1 & o \\
\hline & $\begin{array}{l}\text { Borde } \\
\text { inf. }\end{array}$ & o & -19 & -41 & -54 & $-6 o$ & -62 & o & -4 & -8 & -11 & -12 & -12 & o & o & o & o & o & o \\
\hline
\end{tabular}

(*) Coeficientes máximos a o,5b (parte central) del muro largo para el cálculo de momentos verticales Mx (véase Figura 5)

$\left.{ }^{* *}\right)$ Coeficientes máximos a una altura o,5a del muro largo para el cálculo de momentos horizontales My (véase Figura 5 )

\begin{tabular}{|c|c|c|c|c|c|c|c|c|c|c|c|c|c|c|c|c|c|c|}
\hline & \multicolumn{6}{|c|}{ Coeficientes para Mz } & \multicolumn{6}{|c|}{ Coeficientes para My } & \multicolumn{6}{|c|}{ Coeficientes para Myz } \\
\hline & Esq. & $\begin{array}{l}0,1 c \\
0,9 c\end{array}$ & $\begin{array}{l}0,2 c \\
0,8 c\end{array}$ & $\begin{array}{l}0,3 c \\
0,7 c\end{array}$ & $\begin{array}{l}0,4 c \\
0,6 c\end{array}$ & $\mathbf{0 , 5 c}$ & Esq. & $\begin{array}{l}0,1 C \\
0,9 c\end{array}$ & $\begin{array}{l}0,2 \mathrm{C} \\
0,8 \mathrm{c}\end{array}$ & $\begin{array}{l}0,3 \mathrm{C} \\
0,7 \mathrm{c}\end{array}$ & $\begin{array}{l}0,4 c \\
0,6 c\end{array}$ & $0,5 \mathrm{C}$ & Esq. & $\begin{array}{l}0,1 c \\
0,9 c\end{array}$ & $\begin{array}{l}0,2 \mathrm{c} \\
0,8 \mathrm{c}\end{array}$ & $\begin{array}{l}0,3 \mathrm{C} \\
0,7 \mathrm{c}\end{array}$ & $\begin{array}{l}0,4 c \\
0,6 c\end{array}$ & $0,5 c$ \\
\hline $\begin{array}{l}\text { Borde } \\
\text { sup. }\end{array}$ & o & o & o & o & o & o & o & o & o & o & o & o & 1 & 7 & 9 & 7 & 4 & o \\
\hline $0,9 \mathbf{a}$ & -2 & o & 2 & 4 & 5 & 6 & -11 & -3 & 1 & 3 & 4 & 4 & 1 & 7 & 8 & 6 & 3 & o \\
\hline $\mathbf{0 , 8 a}$ & -4 & o & 5 & 8 & 10 & 11 & -20 & -5 & 2 & 6 & 7 & 7 & 1 & 6 & 7 & 5 & 3 & o \\
\hline $0,7 \mathbf{a}$ & -6 & 1 & 7 & 12 & 15 & 16 & -28 & -7 & 3 & 8 & 10 & 10 & o & 5 & 5 & 4 & 2 & o \\
\hline $0,6 a$ & -7 & 1 & 9 & 14 & 18 & 19 & -34 & -8 & 4 & 10 & 11 & 12 & o & 2 & 2 & 1 & 1 & o \\
\hline $0,5 a$ & -7 & 1 & 10 & 16 & 19 & 21 & -36 & -8 & 5 & 10 & 12 & 12 & o & o & 1 & 1 & 1 & o \\
\hline $0,4 a$ & -7 & 2 & 10 & 15 & 18 & 19 & -34 & -6 & 5 & 9 & 10 & 11 & o & 3 & 4 & 3 & 2 & o \\
\hline $0,3 a$ & -6 & 2 & 7 & 11 & 12 & 13 & -28 & -4 & 4 & 7 & 8 & 8 & o & 6 & 7 & 5 & 3 & o \\
\hline $0,2 a$ & -4 & o & 2 & 1 & 1 & o & -18 & -2 & 2 & 3 & 3 & 3 & 1 & 7 & 8 & 6 & 3 & o \\
\hline $\mathbf{0 , 1 a}$ & -1 & -4 & -10 & -16 & -20 & -21 & -6 & -1 & -1 & -2 & -3 & -3 & 1 & 6 & 6 & 4 & 2 & o \\
\hline $\begin{array}{l}\text { Borde } \\
\text { inf. }\end{array}$ & o & -12 & -31 & -44 & -51 & -53 & o & -2 & -6 & -9 & -10 & -11 & o & o & o & o & o & o \\
\hline
\end{tabular}

Para el cálculo de momentos de diseño, se empleará la expresión dada por la PCA, donde:

$\mathrm{a}=3.00 \mathrm{~m}, \mathrm{~b}=\mathrm{c}=4.80 \mathrm{~m}$ y la carga debido a la presión del agua $\mathrm{q}_{1}=3000 \mathrm{~kg} / \mathrm{m}^{2}$.

$$
\mathrm{Mx}, \mathrm{y}, \mathrm{z}=\operatorname{Coef} \mathrm{x} \frac{\mathrm{q} \mathrm{a}}{1000}=\operatorname{Coef} \mathrm{x} \frac{3000 \times 3^{2}}{1000}=\operatorname{Coef} \mathrm{x} 27 \mathrm{~kg}-\mathrm{m}
$$

El ACI 350 recomienda amplificar los momentos de diseño en estructuras sanitarias, multiplicando por: 1 ) el factor 1,7, por efectos de empuje del agua y 2) el factor de durabilidad sanitaria $S=1,3$; de la siguiente manera:
$\mathrm{Mu}_{\mathrm{x}, \mathrm{y}, \mathrm{z}}=$ coef sanitario $\mathrm{x}$ coef empuje $\mathrm{x} \mathrm{Mx}, \mathrm{y}, \mathrm{z}=1.3 \mathrm{x}$ $1.7 \times 27 \times$ coef $=59.67 \times$ coef

Con esta expresión se determinan los momentos máximos verticales $(\mathrm{Mx})$ y horizontales $(\mathrm{My})$, multiplicando por sus respectivos coeficientes presentados en la Tabla 3; con dichos valores se diseña el acero correspondiente.

En la Tabla 4 se observan momentos verticales en el muro largo (plano X-Y), los cuales son máximos en el borde inferior central para $\mathrm{X}=\mathbf{2 . 4 0} \mathrm{m}$. 
Tabla 4. Momentos Mx para diseño del acero vertical en el muro largo del reservorio para la condición de carga única

\begin{tabular}{|c|c|c|c|c|c|c|}
\hline \multirow{2}{*}{} & \multicolumn{7}{|c|}{ Momentos Mx-Muro largo } \\
\cline { 2 - 6 } $\mathrm{x}$ & 0,00 & 0,48 & 0,96 & 1,44 & 1,92 & \multirow{2}{*}{2,40} \\
\cline { 2 - 6 } $\mathrm{y}$ & 4,80 & 4,32 & 3,84 & 3,36 & 2,88 & 0,00 \\
\hline 3,00 & 0,00 & 0,00 & 0,00 & 0,00 & 0,00 & 477,36 \\
\hline 2,70 & $-119,34$ & 59,67 & 238,68 & 358,02 & 477,36 & 954,72 \\
\hline 2,40 & $-238,68$ & 119,34 & 477,36 & 716,04 & 895,05 & 1312,74 \\
\hline 2,10 & $-358,02$ & 179,01 & 656,37 & 1014,39 & 1253,07 & 1491,75 \\
\hline 1,80 & $-417,69$ & 238,68 & 835,38 & 1253,07 & 1432,08 & $1551,42 *$ \\
\hline 1,50 & $-417,69$ & 298,35 & 895,05 & 1312,74 & 1491,75 & $1551,42 *$ \\
\hline 1,20 & $-417,69$ & 298,35 & 895,05 & 1312,74 & 1491,75 & 775,71 \\
\hline 0,90 & $-358,02$ & 238,68 & 596,70 & 716,04 & 775,71 & $-179,01$ \\
\hline 0,60 & $-238,68$ & 59,67 & 59,67 & $-59,67$ & $-119,34$ & $-1611,09$ \\
\hline 0,30 & $-59,67$ & $-358,02$ & $-895,05$ & $-1312,74$ & $-1551,42$ & $-3699,54$ * \\
\hline
\end{tabular}

(*) Momentos verticales máximos en el muro largo del reservorio

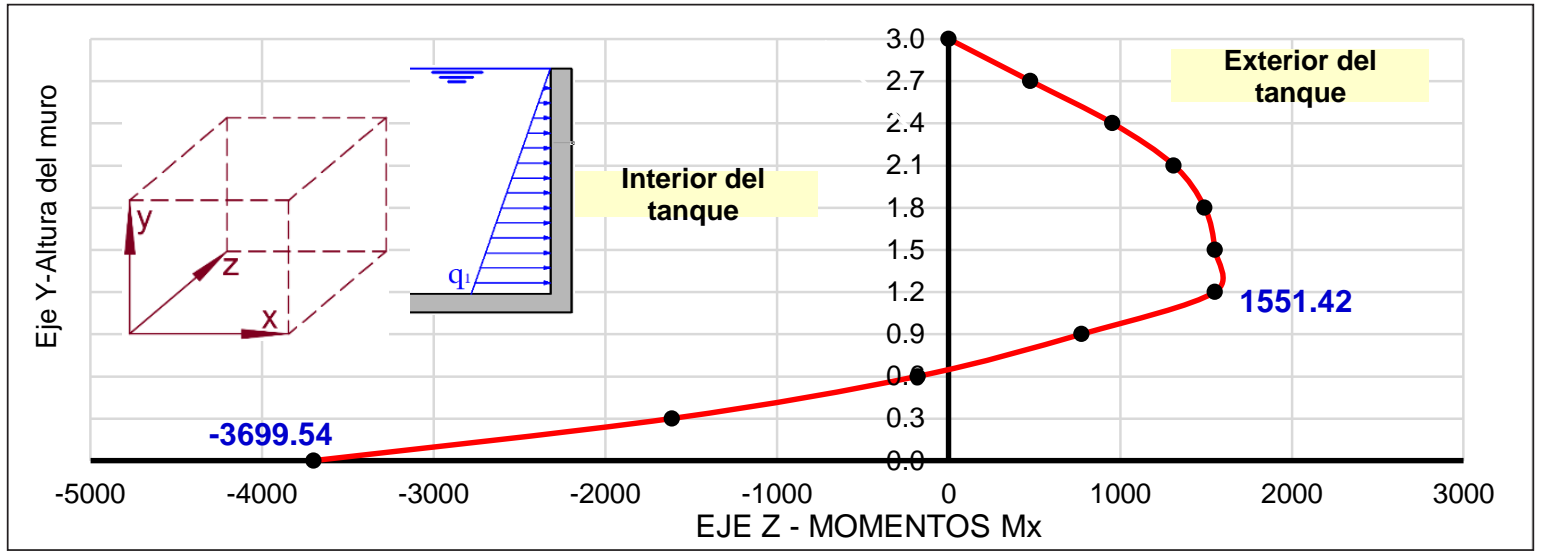

Figura 8. Diagrama de momentos verticales máximos en el muro del reservorio para la condición de carga única

En la Figura 8 se observa que el momento flector en la base del muro tracciona la cara interior en contacto con el agua, y hacia la parte central la tracción por flexión sucede hacia la cara exterior del muro del tanque con un menor valor del que sucede en la base.

Con los valores de momentos máximos de la Figura 8 se diseña el acero vertical, tal y como se muestra en la Tabla 5 .

En la Tabla 6 se observan los momentos horizontales en el muro largo (plano X-Y), los cuales son máximos en los bordes del muro para una altura $\mathrm{Y}=1.50$ m, como se ilustra en la Figura 9.

En la Figura 8 se observa que el momento flector en la base del muro tracciona la cara interior en con- tacto con el agua, y hacia la parte central la tracción por flexión sucede hacia la cara exterior del muro del tanque con un menor valor del que sucede en la base.

Con los valores de momentos máximos de la Figura 8 se diseña el acero vertical, tal y como se muestra en la Tabla 5 .

En la Tabla 6 se observan los momentos horizontales en el muro largo (plano X-Y), los cuales son máximos en los bordes del muro para una altura $Y=1.50$ m, como se ilustra en la Figura 9.

Con los valores de momentos máximos de la Figura 9 se diseña el acero vertical, tal y como se muestra en la Tabla 7. 
Tabla 5. Diseño del acero vertical en el muro largo del reservorio para la condición de carga única

\begin{tabular}{|c|c|c|c|c|}
\hline \multicolumn{5}{|c|}{ Diseño del acero vertical-Muro largo } \\
\hline Base & b & 100 & 100 & $\mathrm{~cm}$ \\
\hline Peralte & h & 30 & 30 & $\mathrm{~cm}$ \\
\hline Peralte efectivo & d & 24 & 24 & $\mathrm{~cm}$ \\
\hline \multirow[t]{2}{*}{ Momento } & Mu & $-3699,54$ & 1551,42 & Kg-m \\
\hline & $\mathrm{ku}$ & 6,42 & 2,69 & \\
\hline Cuantía requerida & $\rho$ & 0,0017 & 0,0011 & \\
\hline \multirow[t]{2}{*}{ Cuantía mínima } & $\rho \min$ & 0,0033 & 0,0033 & \\
\hline & $4 / 3 P_{\text {req }}$ & 0,0023 & 0,0015 & \\
\hline Cuantía seleccionada & $\rho$ & $0,0030^{*}$ & $0,0030^{*}$ & \\
\hline As calculado & As cal & 7,20 & 7,20 & $\mathbf{c m}^{2}$ \\
\hline Refuerzo elegido & & ${ }^{\dagger} 1 / 2 »$ & ${ }^{\dagger} 1 / 2 »$ & \\
\hline Espaciamiento & s & 0,176 & 0,176 & $\mathrm{~m}$ \\
\hline Acero colocado & As col & Ø 1/2»@ 0,175 & $\varnothing_{1 / 2 » @ 0,175}$ & \\
\hline Acero colocado & As col & 7,62 & 7,62 & $\mathrm{~cm}^{2}$ \\
\hline Refuerzo colocado en & & Cara interior del tanque & Cara exterior del tanque & \\
\hline
\end{tabular}

Tabla 6. Momentos My para diseño del acero horizontal en el muro del reservorio para la condición de carga única

\begin{tabular}{|c|c|c|c|c|c|c|}
\hline \multicolumn{7}{|c|}{ Momentos My - Muro largo } \\
\hline & 0,00 & 0,48 & 0,96 & 1,44 & 1,92 & \multirow{2}{*}{2,40} \\
\hline y & 4,80 & 4,32 & 3,84 & 3,36 & 2,88 & \\
\hline 3,00 & 0,00 & 0,00 & 0,00 & 0,00 & 0,00 & 0,00 \\
\hline 2,70 & $-656,37$ & $-59,67$ & 119,34 & 179,01 & 179,01 & 179,01 \\
\hline 2,40 & $-1193,40$ & $-119,34$ & 238,68 & 358,02 & 358,02 & 358,02 \\
\hline 2,10 & $-1670,76$ & $-179,01$ & 358,02 & 477,36 & 477,36 & 477,36 \\
\hline 1,80 & $-2028,78$ & $-179,01$ & 417,69 & 596,70 & 596,70 & 537,03 \\
\hline 1,50 & $-2148,12$ * & $\begin{array}{l}-179,01 \\
\end{array}$ & 477,36 & 596,70 * & 596,70 * & 537,03 \\
\hline 1,20 & $-2028,78$ & $-119,34$ & 417,69 & 537,03 & 477,36 & 477,36 \\
\hline 0,90 & $-1670,76$ & $-59,67$ & 358,02 & 358,02 & 298,35 & 298,35 \\
\hline 0,60 & $-1074,06$ & 0,00 & 179,01 & 119,34 & 59,67 & 59,67 \\
\hline 0,30 & $-358,02$ & $-59,67$ & $-119,34$ & $-238,68$ & $-298,35$ & $-298,35$ \\
\hline 0,00 & 0,00 & $-238,68$ & $-477,36$ & $-656,37$ & $-716,04$ & $-716,04$ \\
\hline
\end{tabular}

(*) Momentos horizontales máximos en el muro largo del reservorio 


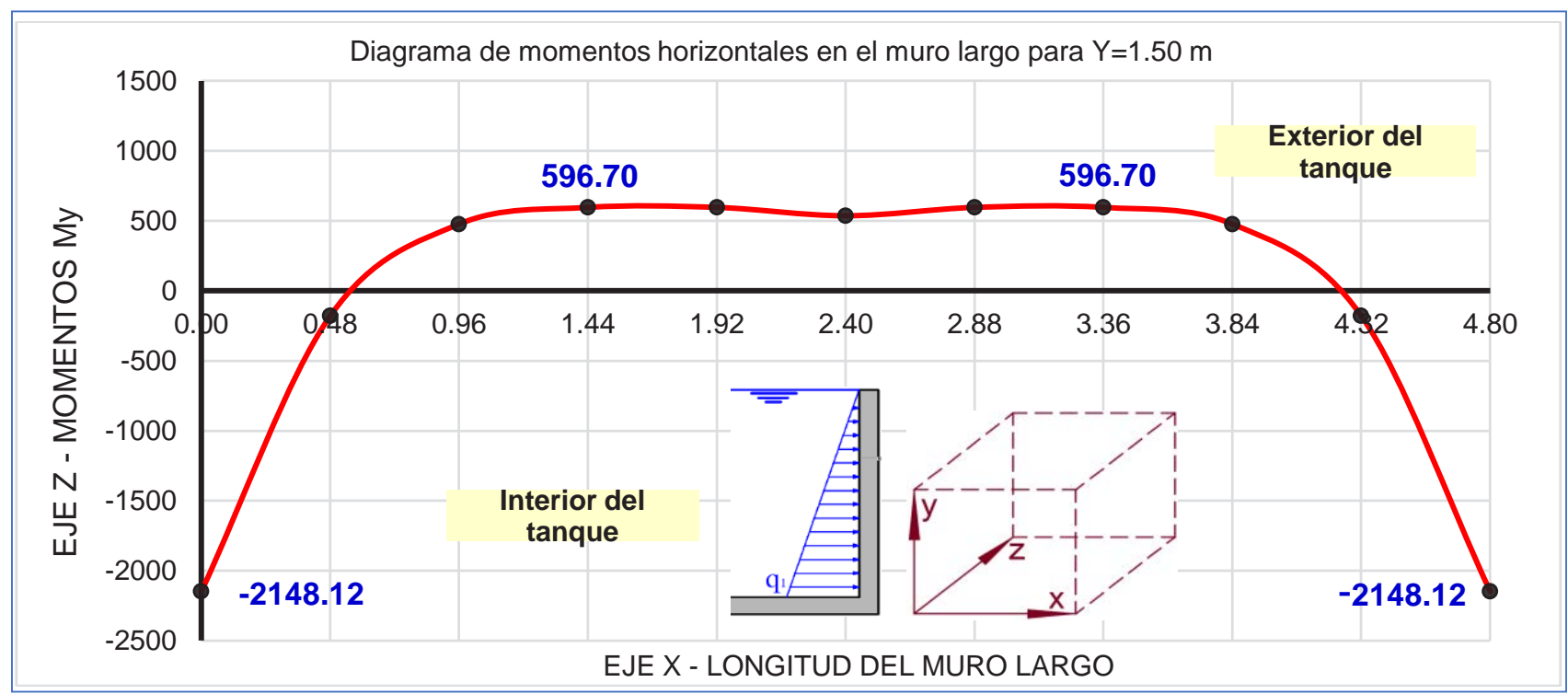

Figura 9. Diagrama de momentos horizontales máximos en el muro largo del reservorio para la condición de carga única

Tabla 7. Diseño del acero horizontal en el muro largo del reservorio para la condición de carga única

\begin{tabular}{|c|c|c|c|c|}
\hline \multicolumn{5}{|c|}{ Diseño del acero horizontal-Muro largo } \\
\hline Base & $\mathrm{b}$ & 100 & 100 & $\mathrm{~cm}$ \\
\hline Peralte & $\mathrm{h}$ & 30 & 30 & $\mathrm{~cm}$ \\
\hline Peralte efectivo & d & 24 & 24 & $\mathrm{~cm}$ \\
\hline \multirow[t]{2}{*}{ Momento } & Mu & $-2148,12$ & 596,70 & kg-m \\
\hline & $\mathrm{ku}$ & 3,73 & 1,04 & \\
\hline Cuantía requerida & $\rho$ & 0,0010 & 0,0003 & \\
\hline \multirow[t]{2}{*}{ Cuantía mínima } & $\rho \min$ & 0,0033 & 0,0033 & \\
\hline & $4 / 3 \rho_{\text {req }}$ & 0,0013 & 0,0004 & \\
\hline Cuantía seleccionada & $\rho$ & 0,0030 * & 0,0030 * & \\
\hline As calculado & As cal & 7,20 & 7,20 & $\mathbf{c m}^{2}$ \\
\hline Refuerzo elegido & & ${ }^{\dagger} 1 / 2 »$ & ${ }^{\dagger} 1 / 2 »$ & \\
\hline Espaciamiento & s & 0.176 & 0,176 & $\mathrm{~m}$ \\
\hline Acero colocado & As col & Ø 1/2»@ 0.175 & Ø 1/2»@ o,175 & \\
\hline Acero colocado & As col & 7,62 & 7,62 & $\mathrm{~cm}^{2}$ \\
\hline Refuerzo colocado en & & Cara interior del tanque & Cara exterior del tanque & \\
\hline
\end{tabular}

* Acero horizontal mínimo de acuerdo al ACI 350, artículo 14.3.3.

† Tamaño mínimo de barra de acero de acuerdo al ACI 350, artículo 7.12.2.2. 


\subsection{Control de fisuras para la condición de carga única}

El control de fisuras se realiza bajo cargas de servicio para verificar el espaciamiento máximo del acero de refuerzo y garantizar la impermeabilidad de las paredes del reservorio.

El máximo momento de flexión vertical empleado en el diseño es Mux = 3699.54 kg-m, cuyo refuerzo calculado es Ø 1/2»@ $0.175 \mathrm{~m}$.

Como el diseño es en la etapa elástica, se determina el máximo momento de flexión en la etapa de servicio:

$$
\mathrm{Ms}=\frac{\mathrm{Mu}}{1.3 \times 1.7}=\frac{3699.54}{1.3 \times 1.7}=1674.00 \mathrm{~kg}-\mathrm{m}
$$

El esfuerzo en el refuerzo calculado para las cargas de servicio es:

As $=7.62 \mathrm{~cm}^{2}$

$\mathrm{d}=30-6=24 \mathrm{~cm}$

$$
\begin{aligned}
& \mathbf{f s}=\frac{\mathbf{M s}}{\mathbf{0 . 9 \mathbf { d ~ A s }}}=\frac{167400}{0.9 \times 24 \times 7.62} \\
& \mathrm{fs}=1017.06 \mathrm{~kg} / \mathrm{cm} 2
\end{aligned}
$$

Según el ACI 350 (Artículo C 10.6.4), fs se puede tomar como el $45 \%$ de fy:

fs $=0.45 \times 4200 \mathrm{~kg} / \mathrm{cm}^{2}=1890 \mathrm{~kg} / \mathrm{cm} 2$

Entonces, podemos trabajar con el promedio:

$(1017.06+1890) / 2=1453,53 \mathrm{~kg} / \mathrm{cm} 2$

El espaciamiento máximo para el control de grietas es:

- Para condiciones severas $\mathrm{z}=17000 \mathrm{~kg} / \mathrm{cm}$ ancho de fisura de $2 \mathrm{~mm}$

- $\mathrm{dc}=\mathrm{r}+\mathrm{db} / 2=5+1,27 / 2=5,63 \mathrm{~cm}$

$$
\begin{gathered}
\text { Smáx }=\frac{\mathbf{0 , 5} \mathbf{z}^{\mathbf{3}}}{{\mathbf{d c ^ { 2 }} \mathbf{f} \mathbf{s}^{\mathbf{3}}}^{\mathbf{2}}=\frac{0,5 \times 17000^{3}}{5,63^{2} \times 1453,53^{3}}} \\
=25 \mathrm{~cm}
\end{gathered}
$$

$25 \mathrm{~cm}>17,5 \mathrm{~cm}$, por lo tanto, el espaciamiento calculado, controla el ancho de las grietas.

\section{CONCLUSIONES}

1. Una manera simple para el análisis y diseño de las paredes con comportamiento bidireccional de los tanques rectangulares es con la ayuda de las tablas de la PCA, Bares, etc.

2. Para el diseño de tanques rectangulares, es importante aplicar las especificaciones del Código Ambiental ACI 350, lo cual se representa en la aplicación del diseño por resistencia en etapa
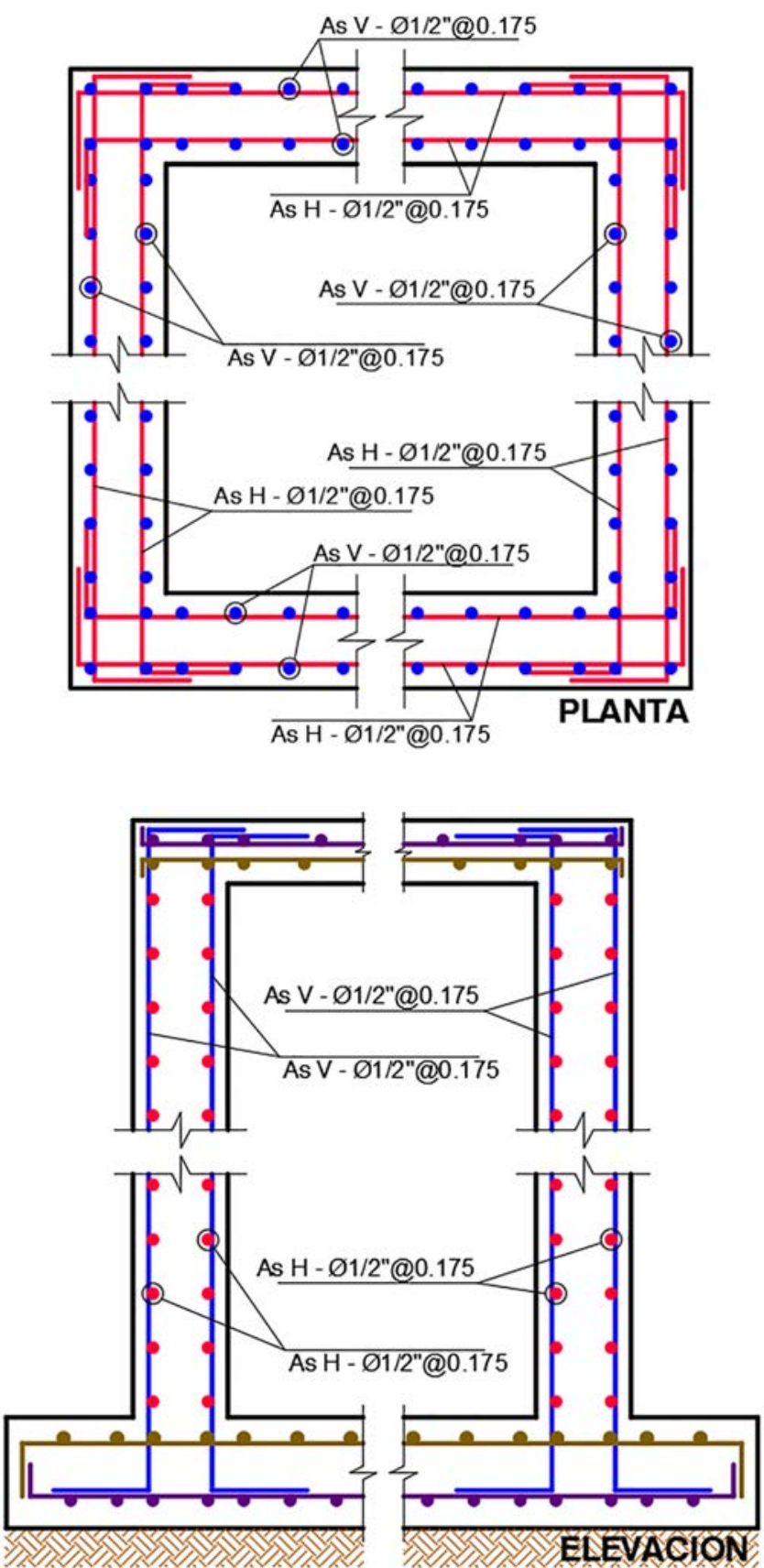

Figura 1o. Detalle del acero en el reservorio

última, amplificado por los coeficientes sanitarios (para flexión, 1,3, y para tracción, 1,65), además de la verificación del acero para el control de fisuras.

3. Un diseño eficiente de los tanques rectangulares que garanticen su resistencia, su durabilidad y la impermeabilidad contribuirá al mejoramiento del medio ambiente.

4. Para tanques superficiales de dimensiones pequeñas, las fuerzas sísmicas no son significativas para el diseño; las fuerzas que prevalecen son las estáticas. 


\section{AGRADECIMIENTOS}

A la Unidad de Investigación de la Facultad de Ingeniería de la Universidad Continental por esta oportunidad.

A Kevin Lavado Sánchez por su apoyo en la traducción del resumen.

\section{BIBLIOGRAFÍA}

[1] MUNSHI, J. Rectangular concrete tanks. Illinois, 5. ${ }^{\mathrm{a}}$ ed. EE.UU.: Portland Cement Association, 1998.

[2] BARES, R. Tablas para el cálculo de placas y vigas pared. Barcelona: Editorial Gustavo Gili, 1970.

[3] ACI Committee 350. Environmental Engineering Concrete Structures (Estructuras sanitarias de concreto para el mejoramiento del ambiente), 2006.

[4] E.o6o Norma Técnica de Concreto Armado. Reglamento Nacional de Edificaciones, 2009.

[5] HARMSEN, T. Diseño de estructuras de concreto armado. 3. ${ }^{\text {a }}$ ed. Lima: Editorial PUPC, 2002.

[6] PAVON, V. Diseño y construcción de estructuras de concreto para contener líquidos. Editorial Fundación ICA-Universidad Autónoma del Estado de México, 2001.

[7] HOUSNER, G. The Dinamic Behavior of Water Tanks, 1963.

[8] ACI 350.3 (Seismic Design of Liquid Containing Concrete Structures (Diseño Sísmico de estructuras contenedoras de líquidos), 2001. 